AperTO - Archivio Istituzionale Open Access dell'Università di Torino

\title{
An analysis of the Podelski-Rybalchenko termination theorem via bar recursion
}

\section{This is a pre print version of the following article:}

Original Citation:

\section{Availability:}

This version is available http://hdl.handle.net/2318/1526156

since 2015-10-07T07:02:17Z

Published version:

DOI:10.1093/logcom/exv058

Terms of use:

Open Access

Anyone can freely access the full text of works made available as "Open Access". Works made available under a Creative Commons license can be used according to the terms and conditions of said license. Use of all other works requires consent of the right holder (author or publisher) if not exempted from copyright protection by the applicable law. 


\section{Effective Bounds on the Podelski-Rybalchenko Termination Theorem}

September 30, 2014

\section{Introduction}

We report here on current work towards an effective proof (with explicit bounds) of Podelski and Rybalchenko Termination Theorem [7]. Our longterm project is to obtain a priori-bounds for the termination of computer programs, and compare these with bounds obtained via other intuitionistic proofs of the Termination Theorem.

Theorem 1.1 (Termination Theorem). A program $P$ is terminating iff there exists a well-founded transition invariant for $P$.

The authors proved this result by using Ramsey Theorem, which is a purely classical results [1]. In [9] and [2] two proofs of an intuitionistic versions of this theorem have been proposed, where the notion of a program being terminating is replaced by intuitionistically weaker (but classically equivalent) notion. Let us call this "inductively terminating".

Theorem 1.2. A program $P$ is (inductively) terminating iff there exists a (inductively) well-founded transition invariant for $P$.

In this result both the hypothesis and the thesis are intuitionistically weaker than the ones in Theorem 1.1, since, intuitionistically, classical wellfoundedness is strictly stronger than intuitionistic well-foundedness. Coquand's proof is based on almost-full relations (see also [4]), while the second one is based on $H$-well-founded relations.

In this work we want to intuitionistically prove Podelski and Rybalchenko Termination Theorem considering the classical definition of well-foundness. In the result by Podelski they define a program to be terminating iff

$$
R \cap(\text { Acc } \times \text { Acc }) \text { is well-founded, }
$$


where $R$ is the transition relation of $P$ and Acc is the set of accessible states. In this work instead we consider the following definition, which is classically equivalent to the Podelski's one. Assume $I$ is any set, $R$ is a binary relation over $I$, and $S$ (the set of initial states) is included in Acc which is included in $I$ (the set of states). We call a computation over $I$ any sequence $\alpha: \mathbb{N} \rightarrow I$, and for all $n \in \mathbb{N}$ we write $\alpha_{n}$ for $\alpha(n)$ in $I$.

Definition 1.3. $P$ is terminating iff $\forall \alpha\left(\alpha_{0} \in S \Longrightarrow \exists n \neg\left(\alpha_{n} R \alpha_{n+1}\right)\right)$.

Lemma 1.4. Classically, $P$ is termimating by Podelski definition iff $P$ is terminating by Definition 1.3 .

Proof. $\Rightarrow$ Assume that $R \cap($ Acc $\times$ Acc $)$ is well founded. Then each chain in the relation $R \cap($ Acc $\times$ Acc $)$ is finite. Moreover every chain which starts with some initial state is finite.

$\Leftarrow$ Assume that

$$
\forall \alpha\left(\alpha_{0} \in S \Longrightarrow \exists n \neg\left(\alpha_{n} R \alpha_{n+1}\right)\right),
$$

we need to prove that each $R \cap(\mathrm{Acc} \times \mathrm{Acc})$-chain is finite. Let $c$ be a chain in $R \cap(\mathrm{Acc} \times \mathrm{Acc})$, then in particular the first element of $c$ is in Acc. Hence we may extend $c$ to a chain $c^{*}$ which start from some initial state. By applying the hypothesis we are done.

Remark 1.5. Note that $\alpha_{0} \in S$ is a decidable formula, whereas $\alpha_{0} \in$ Acc is potentially $\Sigma_{1}$ when $I$ is infinite. Hence, $P$ being terminating is a $\Pi_{2}$ formula, which would not be the case had we used the definition $\forall \alpha\left(\alpha_{0} \in\right.$ Acc $\left.\Longrightarrow \exists n \neg\left(\alpha_{n} R \alpha_{n+1}\right)\right)$.

From now on we will say " $P$ is terminating" meaning it is terminating as in Definition 1.3. We will prove the following result:

Theorem 1.6. Given a program $P$, with transition relation $R$, if

$$
\begin{gathered}
\exists n \exists T_{1}, \ldots, T_{n} \exists \omega_{1}, \ldots, \omega_{n}\left(T_{1} \cup \cdots \cup T_{n} \supseteq R^{+} \cap(\text { Acc } \times \text { Acc })\right. \\
\left.\wedge \forall i \in[1, n]\left(\forall \alpha \exists j<\omega_{i}(\alpha) \neg\left(\alpha_{j} T_{i} \alpha_{j+1}\right)\right)\right)
\end{gathered}
$$

then there exists $\Phi$ such that

$$
\forall \alpha\left(\alpha_{0} \in S \Longrightarrow \exists m<\Phi(\bar{T}, \bar{\omega}) \neg\left(\alpha_{m} R \alpha_{m+1}\right)\right) .
$$


The functional $\Phi$ is definable in $T+$ Bar recursion. Thanks to the results of Schwitchtenberg [8] and Kohlenbach [5] that give some closure properties for bar recursion, we can study the complexity of the bound $\Phi$ relative to the complexity of the relations $T_{i}$ and the functions $\omega_{i}$.

The basic ideas behind the proof are those presented in [6] for the classical Ramsey theorem. Our proof of Theorem 1.6 requires the Transitive Ramsey Theorem for pairs, a corollary of the Ramsey Theorem for pairs which can be stated as follows:

Theorem 1.7 (Transitive Ramsey Theorem for Pairs). For any $c: \mathbb{N}^{2} \rightarrow n$ there exists an infinite homogeneous chain.

The structure of the proof is the following. Given a computation $\alpha$, we consider all initial finite subsequences of $\alpha$. We associate to each initial finite subsequence of $\alpha$ a finite tree which follows the idea of the Blackwell proof of Erdős-Szerkeres Theorem (see [3]). Then from each of these trees we obtain a set of monochromatic sequences whose length is bounded by the $\omega_{i}$. Therefore if $\alpha$ is infinite by applying bar recursion we obtain a contradiction, which gives us the bound we use to define $\Phi$.

\section{Proof for 2 colors}

The goal of this section is to prove Theorem 1.6 for two colors. We first generalize Blackwell proof of Erdős-Szerkeres Theorem in [3] for Transitive Ramsey Theorem. If the coloring is not transitive then we adapt this proof and we build either an infinite homogeneous chain in color 0 or an infinite homogeneous set in color 1 (instead of infinite homogeneous set in both the cases).

Theorem 2.1 (Transitive Ramsey Theorem). Let $c: \mathbb{N}^{2} \rightarrow 2$, then there exists an infinite chain in color 0 or an infinite homogeneous set in color 1 .

Proof. Given a well ordered set $X$ we say that $s$ is a leftmost sequence of $X$ iff all $s_{i} \in X$ and

- $s_{0}=\min X$;

- $s_{i+1}>s_{i}$

- $c\left(\left\{s_{i}, s_{i+1}\right\}\right)=0$

- $\forall x^{X}\left(s_{i}<x<s_{i+1} \Longrightarrow c\left(s_{i}, x\right)=1\right)$ 
Then we construct a set of sequences, as Blackwell does, as follows.

- $w_{0}$ is the leftmost sequence of $\mathbb{N}$.

- $w_{i}$ is the leftmost sequence of $\mathbb{N} \backslash \bigcup\left\{w_{j}: j<i\right\}$.

Since $\mathbb{N}$ is infinite we have either an infinite sequence $w_{i}$ or infinitely many finite non-empty sequences $w_{i}$. In the first case we will have an infinite 0 -chain. In the second case let $\left\{l_{i}: i \in \mathbb{N}\right\}$ be the set of the last element of each such sequence. Since it is an infinite sequence of natural numbers, it should contain an infinite increasing subsequence. This is an infinite 1homogeneous set by construction, since we only ever stop a sequence $w_{i}$ if there are no more 0-edges from the last element of $w_{i}$. In fact the last element $l_{i}$ of each sequence $w_{i}$ is by construction related by a color 1 edge to each element of $\left\{x \in \mathbb{N} \mid x>y \wedge x \notin \bigcup\left\{w_{j} \mid j \leq i\right\}\right\}$. In particular, for all $j>i$ if $l_{j}>l_{i}$, then they are related by one color 1 edge.

Now we can prove Theorem 1.6 for two colors. Let $P$ be a program with its transition relation $R$. Assume $\alpha$ is such that $\alpha_{0} \in S$, where $S$ is the set of the initial states of $P$ and that there exists $T_{0}, T_{1}, \omega_{0}, \omega_{1}$ as in the hypothesis of Theorem 1.6. We have to prove that there exists $\Phi$ such that

$$
\exists m<\Phi(\alpha) \neg\left(\alpha_{m} R \alpha_{m+1}\right) .
$$

The idea is to consider finite approximations of the tree given by the leftmost sequences of the Blackwell proof, and work with these approximations.

In order to do this we need to define the following functions. The first one which we call $\beta$ is the function which gives us the successor (in the sense of the leftmost sequences) of a node with respect to a finite sequence. If a successor does not exists in the given finite sequence $\beta$ returns the empty set.

Definition 2.2. Let $x \in \mathbb{N}$ and let $x_{1}<\cdots<x_{n} \in \mathbb{N}$.

$$
\beta\left(x,\left\langle x_{1}, \ldots, x_{n}\right\rangle\right)=\left\{\begin{array}{cc}
x_{i} \quad \text { if for some } i \in[1, n] \\
\\
x_{i}>x \wedge \\
c\left(\left\{x, x_{i}\right\}\right)=0 \wedge \\
\forall j<i\left(c\left(\left\{x, x_{j}\right\}\right)=1 \vee x_{j}<x\right) \\
\emptyset \quad \text { otherwise. }
\end{array}\right.
$$

Observe that $\beta$ is primitive recursive. Given a non-empty list $l$, we define $\operatorname{hd}(l)$ as the first element of $l, \operatorname{tl}(l)$ as the tail of $l$ and last $(l)$ as the last element of $l$. Now we define a function $\varphi$ which provides the finite approximation of the tree of leftmost sequences. 
Definition 2.3. Let $s$ be a finite list of natural numbers, $\left\langle w_{i}\right\rangle_{i \in \mathbb{N}}$ be a list of finite non-empty lists of natural numbers and let $k \in \mathbb{N}$. Define the function $\varphi$ as

$$
\begin{aligned}
& \varphi(\emptyset, s)= \begin{cases}\emptyset & \text { if } s=\emptyset \\
\varphi(\langle\operatorname{hd}(s)\rangle, \operatorname{tl}(s)) & \text { otherwise }\end{cases} \\
& \varphi\left(\left\langle w_{i}\right\rangle_{i \leq k}, s\right)= \begin{cases}\left\langle w_{i}\right\rangle_{i \leq k} & \text { if } s=\emptyset ; \\
\varphi\left(\left\langle w_{i}\right\rangle_{i<k} *\left(w_{k} *\langle x\rangle\right), s \backslash x\right) & \text { if } \beta\left(\operatorname{last}\left(w_{k}\right), s\right)=x \\
\varphi\left(\left\langle w_{i}\right\rangle_{i \leq k} *\langle\operatorname{hd}(s)\rangle, \operatorname{tl}(s)\right) & \text { if } \beta\left(\operatorname{last}\left(w_{k}\right), s\right)=\emptyset\end{cases}
\end{aligned}
$$

Define $x<_{i} y$ iff $x<y$ and $c(\{x, y\})=i$. Observe that the following are invariants for $\varphi$ :

- for all $i \leq k, w_{i}$ is finite, not empty and is a chain for $<_{0}$.

- for all $i<k$ and $y \in s$, last $\left(w_{i}\right)<y$ implies last $\left(w_{i}\right)<_{1} y$.

- for all $i<j \leq k$, last $\left(w_{i}\right)<\operatorname{last}\left(w_{j}\right)$ implies last $\left(w_{i}\right)<1 \operatorname{last}\left(w_{j}\right)$.

The proof that the first and the second ones are invariant follows from the construction, while the third one holds since if $x>\operatorname{last}\left(w_{j}\right) \wedge x>_{0} \operatorname{last}\left(w_{j}\right)$ then we should have added it in a previous step of the construction, at the end of the $j$-line. So $x$ does not belong to $s$. Moreover, since $s$ is a finite list and its length decreases during the computation of $\varphi, \varphi$ terminates.

The last definition we need is the definition of $\sigma$. Given a sequence of natural numbers $b$, the map $\sigma$ produces an increasing subsequence.

Definition 2.4. Let $b=\left\langle x_{1}, \ldots, x_{n}\right\rangle$ be a list of natural numbers, define $\sigma(b)$ as

$$
\sigma\left(\left\langle x_{1}, \ldots, x_{n}\right\rangle\right)= \begin{cases}\emptyset & \text { if } n=0 \\ \left\langle x_{1}\right\rangle * \sigma\left(\left\langle x_{j}, \ldots, x_{n}\right\rangle\right) & \text { if }\left(x_{j}>x_{1}\right) \wedge \forall h<j\left(x_{h}<x_{1}\right)\end{cases}
$$

Given a list of non-empty finite lists of natural number $\left\langle w_{i}\right\rangle_{i \leq k}$, let us denote by $b$ the list of the last elements of these lists, i.e.

$$
b=\left\langle\operatorname{last}\left(w_{i}\right) \mid i \leq k\right\rangle .
$$

Recall that we are assuming we are giving "moduli of termination" $\omega_{i}$ for each of the relations $T_{i}$. The main property of the modulus is

$$
\forall \alpha \exists j<\omega_{i}(\alpha) \neg\left(\alpha_{j} T_{i} \alpha_{j+1}\right),
$$


i.e. for any given infinite sequence the modulus of termination gives us an upper bound on the point where termination is guaranteed to have happened. Therefore, we must consider a large enough approximation of the Blackwell tree, so as to make sure that we obtain a counter-example on one of the two colours. The following function $\xi$ will check whether it is enough to consider the first $n$ elements of a given sequence $\alpha$, but notice that this function as defined is not primitive recursively. It is essentially performing an unbounded search on $n$. We claim that this can be defined using Spector's bar recursion.

Definition 2.5. Given $\alpha$ and $n$ let $\varphi(\emptyset,\langle 0, \ldots, n\rangle)=\left\langle w_{i}\right\rangle_{i \leq k}$. Define

$$
\begin{aligned}
\xi(\alpha, n) & = \begin{cases}n & \text { if } \exists i \leq k\left(\omega_{0}\left(w_{i}\right)<\left|w_{i}\right|\right) \vee \omega_{1}(\sigma(b))<|\sigma(b)| \\
\xi(\alpha, n+1) & \text { otherwise }\end{cases} \\
\Phi(\alpha) & =\xi(\alpha, 0) .
\end{aligned}
$$

Observe that it depends from $\alpha$ since the coloring (fixed from now on) is defined as follows: $c(i, j)=d$ iff $i<j$ and $\alpha_{i} T_{d} \alpha_{j}$, for each $d \in\{0,1\}$.

\section{$3 \Phi$ is in $\mathrm{T}$}

In this section we prove that $\Phi(\alpha)$ is in T for any computation $\alpha$. Observe that $\Phi(\alpha)$ builds what we call a finite structure $M$ which corresponds to a sequence $\left\langle w_{i}\right\rangle_{i \leq k}$, an approximation of the Blackwell's tree. Firstly we prove that given a sequence $\gamma$ satisfying certain conditions, we can compute the structure $M$ by primitive recursion. Then we will approximate $\gamma$ via bar recursion, and we will prove that such $\gamma$ is in $T$. Therefore also $\Phi(\alpha)$ is.

\subsection{The construction of the structure $M$ given an oracle $\gamma$}

Let $A(i, k, X)$ abbreviate the formula $k>i \wedge c(i, k)=0 \wedge k \notin X$. First, let us assume that we have a sequence $\gamma(X)(i)$ such that for all $i$

$$
\exists k A(i, k, X) \Leftrightarrow A(i, \gamma(X)(i), X) \wedge \forall j<\gamma(X)(i) \neg A(i, j, X)
$$

where $X$ is a finite set of integers. The sequence $\gamma(X)$ is obviously noncomputable as it finds a least witness for $\exists k A(i, k, X)$ whenever such witness exists.

Nevertheless, we now show that given such sequence the construction of the structure $\Phi(\alpha)$ is effective. We will then find an effective approximation to $\gamma$ which will be good enough for ours purposes. 
Let us denote by $\mu_{\mathrm{Sp}}(\omega)(\alpha)$ the primitive recursive functional that finds the first point $n$ such that $\omega(\overline{\alpha, n})<n$. Also, let $\gamma^{k}(X)(i)$ be defined as

$$
\begin{aligned}
& \gamma^{0}(X)(i)=i \\
& \gamma^{k+1}(X)(i)=\gamma\left(X \cup \bigcup_{h \leq k}\left\{\gamma^{h}(X)(i)\right\}\right)\left(\gamma^{k}(X)(i)\right) .
\end{aligned}
$$

Definition 3.1. Define by simultaneous primitive recursion the following sequences:

$$
\begin{array}{ll}
\alpha_{0}=\lambda k \cdot \gamma^{k}(\emptyset)(0) & \alpha_{i+1}=\lambda k \cdot \gamma^{k}\left(X_{i}\right)(0) \\
n_{0}=\mu_{\mathrm{Sp}}\left(\omega_{0}\right)\left(\alpha_{0}\right) & n_{i+1}=\mu_{\mathrm{Sp}}\left(\omega_{0}\right)\left(\alpha_{i+1}\right) \\
w_{0}=\text { maximal 0-prefix of } \alpha_{0} & w_{i+1}=\text { maximal 0-prefix of } \alpha_{i+1} \\
X_{0}=\left\{w_{0}(k)\right\}_{k<\left|w_{0}\right|} & X_{i+1}=X_{i} \cup\left\{w_{i+1}(k)\right\}_{k<\left|w_{i+1}\right|} \\
a_{0}=\mu k\left(k \notin X_{0}\right) & a_{i+1}=\mu k\left(k \notin X_{i+1}\right) \\
j_{0}=\max \left\{\alpha_{0}(k)\right\}_{k \leq n_{0}} & j_{i+1}=\max \left\{\alpha_{i+1}(k)\right\}_{k \leq n_{i+1}}
\end{array}
$$

We explain these as follows: $\alpha_{i}$ is the chain generated by $\gamma$ starting with the smallest number not yet used, i.e. $a_{i-1}$, while avoiding all numbers already used, i.e. $X_{i-1}$. The point $n_{i}$ is the first such that $\omega_{0}\left(\overline{\alpha_{i}, n_{i}}\right)<n_{i}$. By the assumption on $\omega_{0}$, we know that before point $n_{i}$ we will find a 1-link in the sequence $\alpha_{i}$, i.e a point $k$ such that $c\left(\alpha_{i}(k), \alpha_{i}(k+1)\right)=1$. We then take $w_{i}$ to be the maximal 0-chain, and $n_{i}$ is clearly a bound on the length of $w_{i}$. The finite sequences $w_{i}$ will form the rows of the Blackwell matrix. We also keep a record in $j_{i}$ of the largest index used in building $w_{i}$. We will see how this is important later.

Definition 3.2. Recall that $\sigma$ is a functional that computes an increasing subsequence of a given sequence together with the indices filtered out. Hence, given the above define the sequences

$$
\begin{aligned}
& b(i)=\operatorname{last}\left(w_{i}\right) \\
& \left\langle\rho_{0}, \rho_{1}\right\rangle=\sigma(b) \\
& m=\mu_{\mathrm{Sp}}\left(\omega_{1}\right)\left(\rho_{1}\right) \\
& \left.t=\text { maximal 1-prefix of } \rho_{1} \text { (note that }|t| \leq m\right)
\end{aligned}
$$

Hence, we see that the following finite Blackwell matrix (parametrised by $\gamma$ ) is sufficient to obtain a contradiction:

$$
M(\gamma)(i)=w_{i}
$$

where $|M(\gamma)|=\rho_{0}(|t|)$. 


\subsection{Approximating $\gamma$ via bar recursion}

Recall that $A(i, k, X) \equiv k>i \wedge c(i, k)=0 \wedge k \notin X$. Let $q, \omega: \mathbb{N}^{\mathbb{N}} \rightarrow \mathbb{N}$ be such that $q(\gamma)=\omega(\gamma)$ is the greatest element in the finite matrix $M(\gamma)$. Observe that $j_{i}$, as define in the previous section, is needed to define the greatest element of $M(\gamma)$ :

$$
q(\gamma)=\omega(\gamma)=\max \left\{j_{i}: i \leq \rho_{0}(|t|)\right\} .
$$

Our goal is to build a finite approximation to $\gamma$, in the sense that it will only satisfy

$$
\exists k \leq q \gamma A(i, k, X) \Longleftrightarrow A(i, \gamma(X)(i), X) \wedge \forall j<\gamma(X)(i) \neg A(i, j, X)
$$

for all $i \leq \omega \gamma$. But this approximation is clearly enough to give us a proper bound, since we only ever use $\gamma$ up to the point $\omega \gamma$ when building the structure $M(\gamma)$. Let us define the following sequence of selection functions:

$$
\varepsilon_{i}(X)(p)= \begin{cases}k & \exists k \in(i, p(i+1)] \backslash X(c(i, k)=0) \\ i+1 & \forall k \in(i, p(i+1)] \backslash X(c(i, k) \neq 0) .\end{cases}
$$

where $k$ in the first case is taken to be the least one witnessing the formula $\exists k \in(i, p(i+1)] \backslash X(c(i, k)=0)$.

Lemma 3.3. $\varepsilon_{i}(X)$ satisfies, for all $p$

$$
\exists k \leq p(a) A(i, k, X) \Longrightarrow A(i, a, X) \wedge \forall j<a \neg A(i, j, X) .
$$

where $a=\varepsilon_{i}(X)(p)$.

Proof. Assume that $\exists k \in\left(i, p\left(\varepsilon_{i}(X)(p)\right)\right](c(i, k)=0 \wedge k \notin X)$, then we cannot have $\varepsilon_{i}(X)(p)=i+1$, then $\exists k \in(i, p(i+1)](c(i, k)=0 \wedge k \notin X)$, therefore $c\left(i, \varepsilon_{i}(X)(p)\right)=0$.

Then we define

$$
\begin{aligned}
\gamma & =\operatorname{EPS}_{\langle\rangle}^{\omega}(\varepsilon)(q) ; \\
p_{s}(x) & =\overline{\operatorname{EPS}_{s * x}^{\omega}(\varepsilon)}\left(q_{s * x}\right) ;
\end{aligned}
$$

hence thanks to the main theorem for any $n<\omega(\gamma)$

$$
\begin{aligned}
\gamma(X)(n) & =\varepsilon_{[\gamma](n)}(X)\left(p_{[\gamma](n)}\right) \\
q \gamma(X) & =\overline{\varepsilon_{[\gamma](n)}}(X)\left(p_{[\gamma](n)}\right)=p_{[\gamma](n)}\left(\varepsilon_{[\gamma](n)}(X)\left(p_{[\gamma](n)}\right)\right)
\end{aligned}
$$


Since the set $\left(\varepsilon_{i}\right)_{i \in \mathbb{N}}$ is indexed in the natural we have that for all $n \in \mathbb{N}$ :

$$
\varepsilon_{[\gamma](X)(n)}=\varepsilon_{n} .
$$

Therefore $\gamma$ satisfies

$$
\begin{gathered}
\forall i<\omega \gamma(\exists k \in(i, q \gamma](c(i, k)=0 \wedge k \notin X) \Longleftrightarrow \\
(\gamma(X)(i)>i \wedge \gamma(X)(i) \notin X \wedge c(i, \gamma(X)(i))=0)) .
\end{gathered}
$$

In fact let $i<\omega(\gamma)$; if $\exists k \in\left(i, q_{\gamma}\right](c(i, k)=0 \wedge k \notin X)$ holds, then we have

$$
\exists k \in\left(i, p_{[\gamma](i)}\left(\varepsilon_{i}(X)\left(p_{[\gamma](i)}\right)\right](c(i, k)=0 \wedge k \notin X)\right.
$$

and this implies (by construction of the selection functions)

$$
\varepsilon_{i}(X)\left(p_{[\gamma](i)}\right)>i \wedge \varepsilon_{i}(X)\left(p_{[\gamma](i)}\right) \notin X \wedge c\left(i, \varepsilon_{i}(X)\left(p_{[\gamma](i)}\right)\right)=0 .
$$

and so

$$
\gamma(X)(i)>i \wedge \gamma(X)(i) \notin X \wedge c(i, \gamma(X)(i))=0 .
$$

To conclude observe that $\operatorname{EPS}_{\langle\rangle}^{\omega}(\varepsilon)(q)$ is defined by Bar Recursion of type 1 (since $q: \mathbb{N}^{\mathbb{N}} \rightarrow \mathbb{N}$ and $s \in \mathbb{N}^{*}$ ) then, thanks to Schwichtenberg's result [8] and by assuming that $\omega_{0}$ and $\omega_{1}$ are in $T$, we can conclude that $\gamma$ is in $T$ and so also $\Phi(\alpha)$ is.

\section{Proof for $n$ colors}

In this section we prove Theorem 1.6 for $n$ colors, following the argument we use in the case with two colors. Firstly we want to generalize Blackwell proof Theorem 2.1 for $n$-many colors. We may observe that it can be done easily by induction.

Theorem 4.1 (Transitive Ramsey Theorem). Let $c: \mathbb{N}^{2} \rightarrow n$, then there exists an infinite homogeneous chain.

Proof. Proof by induction on $n$. Assume it holds for $n$, then consider a coloring $c: \mathbb{N}^{2} \rightarrow n+1$. Given a well ordered set $X$ we say that $s$ is a leftmost sequence of $X$ iff all $s_{i} \in X$ and

- $s_{0}=\min X$;

- $s_{i+1}>s_{i}$

- $c\left(\left\{s_{i}, s_{i+1}\right\}\right)=0$ 
- $\forall x^{X}\left(s_{i}<x<s_{i+1} \Longrightarrow c\left(s_{i}, x\right) \neq 0\right)$

Then we construct a set of sequences, as Blackwell does, as follows.

- $w_{0}$ is the leftmost sequence of $\mathbb{N}$.

- $w_{i}$ is the leftmost sequence of $\mathbb{N} \backslash \bigcup\left\{w_{j}: j<i\right\}$.

Since $\mathbb{N}$ is infinite we have either an infinite sequence or infinitely many finite sequences. In the first case we will have an infinite 0 -chain and we are done. In the second case let $\left\{l_{i}: i \in \mathbb{N}\right\}$ be the set of the last element of each such sequence. Since it is an infinite sequence of natural numbers, it should contain an infinite increasing subsequence. This is an infinite set in $n$ colors, by construction. Then by induction hypothesis there exists an infinite homogeneous chain.

The idea now is to prove Theorem 1.6 for $n$, by using the induction on $n$ and the schema of the proof for two colors. Assume that the Theorem 1.6 holds for $n$, we will prove it for $n+1$.

Let $P$ be a program with its transition relation $R$. Assume $\alpha$ is such that $\alpha_{0} \in S$, where $S$ is the set of the initial states of $P$ and that there exist $T_{0}, \ldots, T_{n}, \omega_{0}, \ldots, \omega_{n}$ as in the hypothesis of Theorem 1.6 .

We have to prove that there exists $\Phi_{n}$ such that

$$
\exists m<\Phi_{n}(\alpha)\left(\neg\left(\alpha_{m} R \alpha_{m+1}\right)\right) .
$$

In order to do this we need to define the following functions as we did in the case for 2 colors.

Definition 4.2. Let $x \in \mathbb{N}, x_{1}<\cdots<x_{m} \in \mathbb{N}$.

$$
\beta_{n}\left(x,\left\langle x_{1}, \ldots, x_{m}\right\rangle\right)=\left\{\begin{array}{cc}
x_{i} \quad & \text { if for some } i \in[1, m] \\
& x_{i}>x \wedge \\
& c\left(\left\{x, x_{i}\right\}\right)=0 \wedge \\
& \forall j<i\left(c\left(\left\{x, x_{j}\right\}\right) \neq 0 \vee x_{j}<x\right) \\
\emptyset \quad \text { otherwise. }
\end{array}\right.
$$

Now we define a function $\varphi_{n}$ (as $\varphi$ for the case with 2 colors) which provides the finite approximation of the tree of leftmost sequences.

Definition 4.3. Let $s$ be a list of natural numbers, $\left\langle w_{i}\right\rangle_{i<\mathbb{N}}$ be a list of lists of finite non-empty lists of natural numbers and let $k \in \mathbb{N}$. Define the function $\varphi_{n}$ as 


$$
\begin{aligned}
& \varphi_{n}(\emptyset, s)= \begin{cases}\emptyset & \text { if } s=\emptyset ; \\
\varphi_{n}(\langle\operatorname{hd}(s)\rangle, \operatorname{tl}(s)) & \text { otherwise. }\end{cases} \\
& \varphi_{n}\left(\left\langle w_{i}\right\rangle_{i \leq k}, s\right)= \begin{cases}\left\langle w_{i}\right\rangle_{i \leq k} & \text { if } s=\emptyset ; \\
\varphi_{n}\left(\left\langle w_{i}\right\rangle_{i<k} *\left(w_{k} *\langle x\rangle\right), s \backslash x\right) \text { if } \beta_{n}\left(\operatorname{last}\left(w_{k}\right), s\right)=x ; \\
\varphi_{n}\left(\left\langle w_{i}\right\rangle_{i \leq k} *\langle\operatorname{hd}(s)\rangle, \operatorname{tl}(s)\right) & \text { if } \beta_{n}\left(\operatorname{last}\left(w_{k}\right), s\right)=\emptyset .\end{cases}
\end{aligned}
$$

While the $\sigma$ is exactly the function defined for the case 2. As in the case for two colors, given a sequence of natural numbers $b$, the map $\sigma$ produces an increasing subsequence.

Definition 4.4. Given $\alpha$ and $n$ let $\varphi(\emptyset,\langle 0, \ldots, m\rangle)=\left\langle w_{i}\right\rangle_{i \leq k}$. Define

$$
\begin{aligned}
& \xi_{n}(\alpha, m)= \begin{cases}m & \text { if } \exists i \leq k\left(\omega_{0}\left(w_{i}\right)<\left|w_{i}\right|\right) \\
& \vee \Phi_{n-1}(\sigma(b))<|\sigma(b)| \\
\xi_{n}(\alpha, m+1) & \text { otherwise }\end{cases} \\
& \Phi_{n}(\alpha)=\xi_{n}(\alpha, 0) .
\end{aligned}
$$

In order to justify the previous definition, observe that in this construction the sequence $\sigma(b)$ is an homogeneous set in $n$ colors, then we are in the following case:

$$
T_{1} \cup \cdots \cup T_{n} \supseteq(R \uparrow \sigma(b))^{+} \cap(\text { Acc } \times \text { Acc }) \wedge T_{1}, \ldots, T_{n} \text { well founded }
$$

Then $R \uparrow \sigma(b)=\sigma(b)$ is well founded (with modulus $\Phi_{n-1}$ ).

Observe that again the definition of $\Phi_{n}$ is not primitive recursive. But we can prove it is in $\mathrm{T}$, by using the same argument we used in the case with two relations.

Proposition 4.5. $\Phi_{n}$ is in $\mathrm{T}$.

Proof. Again by induction. For $n=2$ we proved it in the previous section. Assume that $\Phi_{n-1}$ is in T. Then, by applying the same procedure we used for the case $n=2$ providing to put $\omega_{1}=\Phi_{n-1}$, we obtain that also $\Phi_{n}$ is in $T$.

\section{References}

[1] Stefano Berardi and Silvia Steila. Ramsey Theorem for pairs as a classical principle in Intuitionistic Arithmetic. submitted, 2013. 
[2] Stefano Berardi and Silvia Steila. Ramsey Theorem as an intuitionistic property of well founded relations. submitted, 2014.

[3] Paul Blackwell. An Alternative Proof of a Theorem of Erdos and Szekeres. The American Mathematical Monthly, 78(3):p. 273, 1971.

[4] Thierry Coquand. A direct proof of Ramsey's Theorem. Author's website, revised in 2011, 1994.

[5] Ulrich Kohlenbach. On the no-counterexample interpretation. J. Symb. Log., 64(4):1491-1511, 1999.

[6] Paulo Oliva and Thomas Powell. A Constructive Interpretation of Ramsey's Theorem via the Product of Selection Functions. Mathematical Structures in Computer Science. to appear.

[7] Andreas Podelski and Andrey Rybalchenko. Transition invariants. In LICS, pages 32-41, 2004.

[8] Helmut Schwichtenberg. On bar recursion of types 0 and 1. J. Symb. Log., 44(3):325-329, 1979.

[9] Dimitrios Vytiniotis, Thierry Coquand, and David Wahlstedt. Stop when you are almost-full - adventures in constructive termination. In ITP, pages 250-265, 2012. 